\title{
Lutter contre la tuberculose chez les Inuit au Canada
}

\author{
M Patterson ${ }^{1 *}$, S Finn ${ }^{1}, K^{2}$ Barker $^{1}$
}

\section{Résumé}

Le taux annuel moyen de tuberculose chez les Inuit au Canada est maintenant plus de 290 fois plus élevé que chez les non-Autochtones nés au Canada. Comment est-ce arrivé? En prenant comme exemple le territoire du Nunavut, les causes profondes de cette situation peuvent être attribuées en grande partie aux déterminants sociaux de la santé et aux difficultés d'accès aux soins de santé. La moitié (52 \%) de tous les résidents du Nunavut vivent dans des logements sociaux, qui sont souvent surpeuplés. Beaucoup sont confrontés à l'insécurité alimentaire, les prix des aliments au Nunavut étant deux fois plus élevés que ceux du Sud du Canada, et $60 \%$ des résidents du Nunavut sont fumeurs. Parmi les difficultés liées à la prestation des soins de santé, mentionnons notamment le fait qu'il s'agit de petites collectivités isolées, avec peu de routes et des conditions météorologiques difficiles pendant les longs hivers, ce qui nuit à la capacité de s'y rendre ou d'y fournir des soins de santé; le personnel qui a peu d'expérience de la tuberculose ou qui est peu sensibilisé à la culture; les multiples besoins concurrents en matière de soins de santé; les ressources limitées et le roulement élevé de personnel. La pénurie de logements n'est pas seulement un déterminant social de la santé; elle a aussi une incidence sur la capacité d'embaucher du nouveau personnel ou d'organiser une intervention efficace en cas d'éclosion.

Pourtant, malgré ces défis, des progrès ont été réalisés. La prise en charge de la tuberculose au Nunavut inclut la recherche active de cas, la localisation des contacts pour tous les cas contagieux de tuberculose et le dépistage chez les enfants d'âge scolaire. Des tests rapides avec la plateforme GeneXpert ${ }^{\odot}$ ont permis de diagnostiquer plus rapidement la tuberculose active, $d^{\prime}$ administrer un traitement plus tôt (ce qui permet de limiter la progression de la maladie) et de réduire la transmission. Les radiographies numériques ont progressivement remplacé les clichés simple, ce qui a permis de réduire le délai d'exécution des radiographies, qui est passé de deux à trois semaines à seulement un ou deux jours. Les protocoles de traitement standards incluent une quadruple thérapie en attendant les résultats de l'antibiogramme, le recours à I'isolement à domicile pour les cas actifs et la thérapie sous observation directe (TOD), aussi bien pour les cas d'infection tuberculeuse latente que pour ceux de tuberculose active. L'accès spécial à la rifapentine (Priftin) et son utilisation en polythérapie (3HP) ne nécessite qu'une seule administration par semaine pendant 12 semaines ou visites, comparativement à 78 visites pour I'isoniazide (INH) ou 120 visites pour la rifampicine, ce qui améliore le taux d'observance et réduit grandement les ressources de santé nécessaires pour traiter la tuberculose.

En octobre 2017, I'honorable Jane Philpott, alors ministre de la Santé et maintenant ministre des Services aux autochtones, et Natan Obed, président de I'Inuit Tapiriit Kanatami (ITK), ont annoncé la création d'un groupe de travail chargé d'élaborer un cadre d'action pour l'élimination de la tuberculose chez les Inuit, qui doit être accompagnée de plans d'action régionaux. On espère que le groupe de travail et les efforts actuels au Nunavut mèneront aux changements à long terme nécessaires pour finalement éliminer la tuberculose chez les Inuit du Canada.

\section{Affiliation}

${ }^{1}$ Ministère de la Santé, gouvernement du Nunavut, lqaluit (Nunavut)

*Correspondance : mpatterson@ gov.nu.ca

Citation proposée : Patterson M, Finn S, Barker K. Lutter contre la tuberculose chez les Inuit au Canada. Relevé des maladies transmissibles au Canada. 2018;44(3/4):92-5. https://doi.org/10.14745/ccdr.v44i34a02f

\section{Introduction}

La tuberculose (TB) est un problème qui perdure pour les peuples autochtones du Canada, en particulier chez les Inuit. Le taux annuel moyen de TB chez les Inuit au Canada est maintenant plus de 290 fois plus élevé que chez les non-Autochtones nés au Canada (1). Cette statistique est manifeste dans le territoire du Nunavut, qui compte une population d'environ 38000 personnes, dont $85 \%$ d'Inuit. Le nombre de cas en 2017 devrait être le plus élevé depuis
2010, année où a été recensé un sommet de 100 cas. Selon les données préliminaires pour 2017, 100 nouveaux cas de TB active, au moins 300 cas de TB latente et deux décès ont été diagnostiqués, sur une population d'environ 38000 personnes. Soixante-huit pour cent $(n=17)$ des 25 collectivités du Nunavut ont déclaré au moins un cas de TB latente ou active (M Patterson, données non publiées, 2017). 
Les terres traditionnelles des Inuit au Canada (I'Inuit Nunangat) sont actuellement divisées en quatre régions : le Nunatsiavut, le Nunavik, le Nunavut et l'Inuvialuit (figure 1). Le Nunavut est la seule partie du Nunangat qui, en tant que territoire canadien, profite d'une autonomie gouvernementale complète. Le Nunavut représente environ $20 \%$ de la masse terrestre du Canada et $0,01 \%$ de sa population. Toutes les personnes qui résident à temps plein dans la région vivent dans l'une de ses 25 collectivités. Bien qu'il y ait d'importantes différences dans l'état de santé des résidents du Nunangat, il y a aussi des traits communs aux quatre régions : l'espérance de vie y est inférieure à la moyenne canadienne; il y a un manque de logement; l'insécurité alimentaire y est répandue; et le taux de chômage y est plus élevé que la moyenne nationale.

\section{Figure $1:$ Les quatre régions englobant les terres traditionnelles des Inuit au Canada (2)}

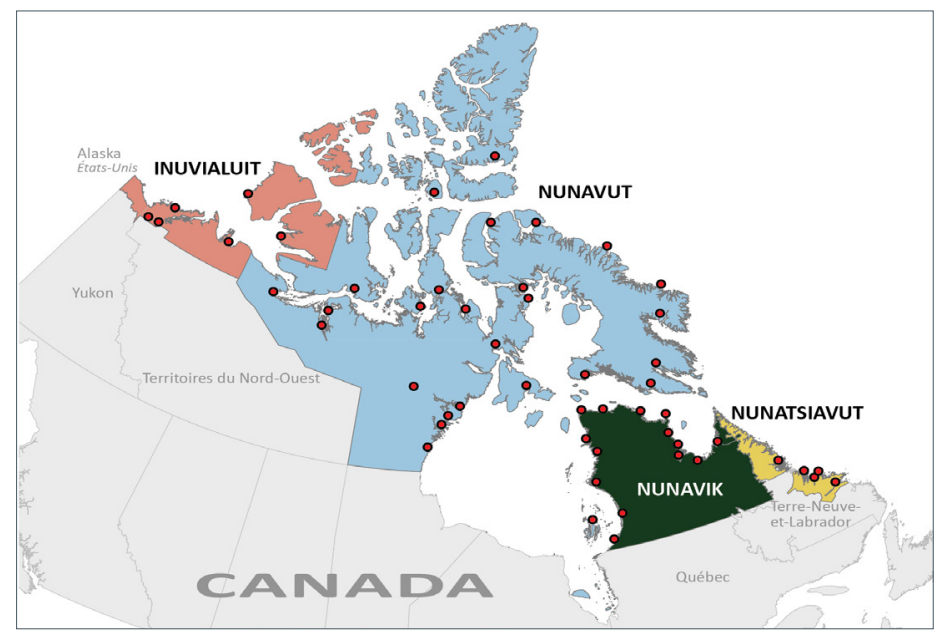

\section{Contexte}

Les tentatives précédentes d'éradication de la TB dans I'Inuit Nunangat ont échoué, car elles étaient minées par des mesures de santé qui n'étaient ni culturellement appropriées ni durables. Dans les années 1950, par exemple, les soins antituberculeux consistaient à transporter les personnes atteintes de TB active vers des sanatoriums dans le Sud, généralement pendant des années, et beaucoup ne revenaient jamais (3). La plupart des Inuit ont des proches qui sont disparus dans ce processus et ils ne sont même pas en mesure de savoir exactement quand et où ils sont morts ou même, dans certains cas, où ils ont été enterrés (4). À partir des années 1970, une campagne de traitement de masse a été menée, dans le cadre de laquelle des fournisseurs de soins de santé visitaient les collectivités, procédaient au dépistage de masse et offraient un traitement à tous ceux qui étaient atteints de TB (3). Lorsque les taux de TB ont diminué, ces efforts ont été abandonnés. Dans les dix à vingt ans après la fin des campagnes de dépistage et de traitement dans les collectivités, les taux de TB ont recommencé à augmenter pour tous les Inuit canadiens. On a donc vu par le passé des efforts significatifs de lutte contre la TB lorsque les taux étaient élevés, suivis d'un déclin des efforts lorsque les taux diminuaient. C'est pourquoi la baisse soutenue des taux de TB chez les Inuit ne s'est jamais concrétisée.
Cet article met l'accent sur le Nunavut à titre d'exemple, car la population de ce territoire est composée à majorité d'Inuit et on y retrouve le taux le plus élevé de TB au Canada. On y décrit les causes profondes des taux élevés de TB au Nunavut, ainsi que les efforts en cours pour améliorer les soins antituberculeux; ces descriptions reflètent en partie ce qui se passe dans d'autres régions de l'Inuit Nunangat et ailleurs au Canada.

\section{Comprendre les causes profondes}

Les causes profondes des taux élevés de TB chez les Inuit prennent racine dans les déterminants sociaux de la santé et dans l'accès inéquitable aux soins de santé.

\section{Déterminants sociaux}

En ce qui concerne les déterminants sociaux de la santé, l'espérance de vie des Inuit (aussi appelés les Nunavummiut dans leur langue, l'inuktitut) est d'environ 10 ans de moins que la moyenne canadienne. Pour les résidents du Nunavut, particulièrement les Inuit, le manque de logements adéquats et I'insécurité alimentaire demeurent des problèmes importants. La moitié (52\%) de tous les Nunavummiut vivent dans des logements sociaux et, dans certaines collectivités, jusqu'à $72 \%$ d'entre eux vivent dans des logements surpeuplés (5). Un logement peut être si bondé que certains dorment en alternance; trop souvent, ils peuvent être plus de 20 personnes à demeurer dans une maison ne comptant que quatre chambres à coucher (5). En outre, de nombreux Nunavummiut vivent en situation d'insécurité alimentaire. Les prix des aliments au Nunavut sont en moyenne deux fois plus élevés que ceux du Sud du Canada (6). Il y a aussi souvent un manque de variété dans les aliments, ce qui peut être accentué encore davantage par les difficultés qui surviennent inévitablement dans le transport par avion de nourriture sur de longues distances.

Contrairement à de nombreux autres groupes autochtones au Canada, I'usage du tabac n'occupait pas une place importante dans la culture inuit avant les premiers contacts avec les cultures européennes. Depuis, le tabagisme a toutefois augmenté au point où au moins $61 \%$ des Nunavummiut fument la cigarette (7), une situation qui, à elle seule, augmente les risques d'infections des voies respiratoires, notamment la TB.

\section{Accès aux soins de santé}

De nombreuses difficultés nuisent à la prestation des soins de santé au Nunavut. La plupart des Inuit vivent dans de petites collectivités côtières éloignées. II n'y a pas de liaisons routières au Nunavut, créant ce qu'on a appelé la «tyrannie de la distance » (8), qui affecte tous les aspects des soins de santé. De plus, les hivers longs et rigoureux, au cours desquels certaines collectivités vivent constamment dans l'obscurité pendant plusieurs semaines ou plus, rendent d'autant plus difficiles l'accès aux services de santé ou la prestation de ceux-ci. Par ailleurs, la plupart des membres du personnel de santé ont été formés dans le Sud du Canada, où ils n'ont peut-être jamais vu de cas de TB. Lorsque des travailleurs de la santé arrivent du Sud pour la première fois, ils ne connaissent souvent pas la langue et la culture locales, ce qui rend difficile la communication avec les Nunavummiut. 
Chez les Inuit, beaucoup de problèmes de santé urgents doivent être gérés avec des ressources de santé limitées. Ainsi, bien qu'il soit impératif de lancer des initiatives de prévention et de lutte contre la TB, il est important que ces efforts n'entraînent pas une diminution des autres initiatives de santé; en réaffectant du personnel actuel vers les mesures de lutte contre la TB, on créerait des vulnérabilités dans d'autres secteurs de la santé. Le manque d'espace adéquat, tant pour le travail que pour le logement, constitue aussi un obstacle à l'amélioration de la prestation des soins antituberculeux au Nunavut. Les pénuries de logements dans de nombreuses collectivités nuisent à la capacité du Nunavut à embaucher du nouveau personnel et à intensifier efficacement les interventions en cas d'éclosion.

\section{Situation actuelle au Nunavut}

Malgré les difficultés, le Nunavut a réalisé certains progrès dans I'amélioration des soins contre la TB. Les pratiques actuelles en matière de traitement de la TB au Nunavut comprennent I'isolement à domicile pour les cas actifs et les traitements sous observation directe (TOD) pour tous les aspects des traitements antituberculeux (TB latente et active). On cherche activement les cas (notamment par la localisation des contacts) pour tous les cas de TB infectieuse, et les enfants d'âge scolaire (de la maternelle à la sixième année) font l'objet d'un dépistage.

Les schémas thérapeutiques actuels pour la TB active comprennent une quadruple thérapie (rifampicine, isoniazide [INH], pyrazinamide et éthambutol) pour la plupart des cas, jusqu'à ce que les sensibilités à ces médicaments contre la TB soient confirmées. Cette approche se justifie par le fait qu'il y a eu peu de cas de résistance à l'INH au Nunavut et que, lors de la plupart des éclosions, bon nombre des personnes infectées ont eu plusieurs contacts pouvant être des sources potentielles, ce qui fait qu'il est impossible de connaître leur véritable source avec certitude.

\section{Progrès récents}

En avril 2017, on a embauché à temps plein une infirmière enseignante spécialiste de la TB, ce qui a amélioré la capacité à former de nouveaux employés aux soins contre la TB. Les fournisseurs de soins qui n'ont pas d'expérience dans les traitements antituberculeux ont l'occasion de passer plusieurs jours à lqaluit pour participer à des activités d'apprentissage didactique sur la TB et se familiariser avec le programme de lutte contre la TB du Nunavut.

À l'automne 2017, Santé Canada a approuvé l'ajout de la rifapentine (RPT) à la Liste des drogues utilisées pour des besoins urgents en matière de santé publique. Auparavant, des recherches ont été menées à Ottawa et à lqaluit sur un traitement de 12 doses hebdomadaires de RPT plus INH (3HP), administré en thérapie sous observation directe pour le traitement des infections tuberculeuses latentes (9). L'avantage du 3HP pour les patients et pour le système de santé est qu'il ne nécessite qu'une visite hebdomadaire pendant 12 semaines, au lieu des 78 visites requises pour I'INH ou des 120 visites pour la rifampicine. Ce traitement sera probablement mieux accepté et plus facile à mener à terme pour les personnes ayant une infection tuberculeuse latente, en plus de réduire considérablement les ressources de santé requises pour traiter la TB.
Depuis 2012, on emploie au Nunavut un test de diagnostic rapide de la TB, le test Xpert $\mathrm{MTB}^{\mathrm{R}} \mathrm{RF}^{\odot}$ (de la société Cepheid inc., à Sunnyvale, en Californie), qui repose sur la technologie de réaction en chaîne de la polymérase (PCR) automatisée et nichée dans une cartouche et qui s'effectue en temps réel au moyen de la plateforme GeneXpert ${ }^{\odot}$. Faisant initialement partie d'un programme de recherche, ce test est maintenant largement utilisé pour tester les expectorations de nombreux résidents de l'Est du Nunavut afin de déceler la présence de la TB. II a été démontré qu'un diagnostic plus précoce de la TB active entraîne un traitement plus rapide (ce qui permet d'empêcher la progression de la maladie) et une diminution de la transmission de la TB (10). Ces résultats peuvent se traduire par des gains importants, tant pour les patients que pour le système de soins de santé.

D'autres efforts en cours pour renforcer le système de santé dans son ensemble au Nunavut ont également permis d'améliorer les soins antituberculeux. L'exemple le plus évident est lié à la mise en place des appareils de radiographie numérique. Jusqu'à assez récemment, la plupart des collectivités du Nunavut utilisaient des radiographies conventionnelles, sur clichés simples. Après le développement de la pellicule dans la collectivité, les clichés étaient envoyés à des radiologues du Sud du Canada aux fins d'interprétation. Le simple fait de passer des clichés sur pellicules aux clichés numériques a permis de réduire le temps d'exécution des radiographies de deux ou trois semaines à un ou deux jours. On espère que d'ici la fin de 2018, la totalité des 25 collectivités du Nunavut auront achevé la transition vers les appareils de radiographie numérique. En outre, le ministère de la Santé du Nunavut a mis en place un programme établi d'abandon de la nicotine qui met l'accent sur l'éducation et le soutien afin d'encourager tous les Nunavummiut à cesser de fumer.

\section{Discussion}

Les taux de TB sont élevés chez les Inuit au Canada, et le Nunavut en est un parfait exemple. Cette situation perdure depuis longtemps, alors que les déterminants sociaux de la santé et l'accès difficile aux soins de santé continuent d'y contribuer. Mais la situation progresse. On a intégré les avancées dans la détection, le diagnostic et le traitement de la TB dans les soins contre la TB dans le Nord. II est possible que I'augmentation récente des taux de TB soit en partie attribuable à une détection accrue. En soutenant les efforts, ces taux devraient ensuite commencer à baisser.

\section{Prochaines étapes}

Compte tenu des taux élevés de TB au Nunavut et dans I'ensemble de l'Inuit Nunangat, on a tenu une réunion pour discuter des problèmes, du 4 au 6 octobre 2017 à Ottawa (11). La réunion était organisée conjointement par le gouvernement du Nunavut et I'Inuit Tapiriit Kanatami (ITK), un organisme national représentant tous les Inuit du Canada. I'Inuit Tapiriit Kanatami (ITK) mène notamment des activités d'éducation et de plaidoyer pour la recherche touchant les Inuit du Canada (12). La rencontre a regroupé des représentants des gouvernements fédéral, provinciaux et territoriaux, des spécialistes cliniques de la TB et des chercheurs pour échanger des idées et des stratégies sur les façons d'améliorer les soins antituberculeux pour tous les Inuit. 
À la fin de cette réunion mixte ITK-Nunavut, I'honorable Jane Philpott, ex-ministre de la Santé et maintenant ministre des Services aux autochtones, et Natan Obed, président d'ITK, ont annoncé la création d'un groupe de travail chargé d'élaborer un cadre d'action pour l'élimination de la TB chez les Inuit, qui doit être accompagné de plans d'action régionaux.

La planification régionale est un volet important. Bien que les causes profondes de l'augmentation des taux de TB soient très similaires dans l'ensemble du Nunangat, il existe des différences importantes entre les régions quant aux défis à surmonter et aux solutions qu'il faut adopter dans la lutte contre la TB. Les systèmes de santé de chaque région sont régis par des contextes réglementaires très différents et doivent composer avec des difficultés logistiques diversifiées. Ainsi, même si chaque région a le même objectif ultime qui consiste à éliminer la TB, les programmes régionaux adopteront des approches qui peuvent différer grandement les unes des autres.

\section{Conclusion}

Avec les taux actuels de TB au Nunavut et dans le Nunangat, il est essentiel de mettre en place un programme robuste et durable de lutte contre la TB sur tout le territoire. Par le passé, on a eu tendance à faire des efforts importants pour améliorer les statistiques lorsque la TB atteignait des sommets, puis à reculer lorsque le nombre de cas diminuait. Pour que les taux de TB diminuent de façon durable, il faut affecter du personnel réservé à la $T B$, et ce, non seulement à court terme, mais aussi pour les années à venir. On espère que les efforts actuels au Nunavut et ceux entrepris par le groupe de travail sur la TB entraîneront les changements à long terme nécessaires pour finalement éliminer la TB chez les Inuit au Canada.

\section{Conflit d'intérêt}

Aucun.

\section{Remerciements}

Nous tenons à remercier toutes les personnes qui ont travaillé d'arrache-pied pour lutter contre la TB dans la population inuit.

\section{Références}

1. Vachon J, Gallant V, Siu W. La tuberculose au Canada, 2016. Relevé des maladies transmissibles au Canada, 2018;44(3/4):85-91. https://www.canada.ca/fr/ sante-publique/services/rapports-publications/releve-maladie s-transmissibles-canada-rmtc/numero-mensuel/2018-44/ numero-3-4-1-mars-2018/article-1-tuberculose-2016.html

2. Statistiques Canada. Carte 1: Les quatre régions inuit du Canada [Consulté le 6 fév 2018]. https://www.statcan.gc.ca/ pub/89-644-x/2010001/m-c/11281/m-c/m-c1-fra.htm
3. Orr P. Tuberculosis in Nunavut: looking back, moving forward. CMAJ 2013 Mar;185(4):287-8. http://dx.doi. org/10.1503/cmaj.121536. PubMed (https://www.ncbi.nlm. nih.gov/entrez/query.fcgi?cmd=Retrieve \&db=PubMed\&lis t_uids=23382256\&dopt=Abstract).

4. Selway S. Nobody Here Will Harm You: Mass Medical Evacuation from the Eastern Arctic 1950-1965. Hamilton, Ontario: James Street North Books; 2016.

5. Nunavut Housing Corporation. Nunavut is facing a severe housing crisis, Nunavut Housing Corporation's Appearance before the Standing Senate Committee on Aboriginal Peoples - March 23, 2016. Iqaluit (NU): Government of Nunavut; 2016 [Consulté le 6 fév 2018]. http://assembly.nu.ca/sites/ default/files/TD\%20158-4(3)\%20EN\%20Nunavut\%20is\%20 Facing\%20a\%20Severe\%20Housing\%20Crisis.pdf

6. Nunavut Bureau of Statistics. StatsUpdate: 2017 Nunavut Food Price Survey. Iqaluit (NU): Government of Nunavut; July 2017 [Conutlé le 6 fév 2018]. http://www.stats.gov. nu.ca/Publications/Historical/Prices/Food\%20Price\%20 Survey,\%20Select\%20ltems\%20Comparison\%20Nunavut_ CanadaCPI\%20StatsUpdate,\%202017.pdf

7. Enquête sur la santé dans les collectivités canadiennes de 2015 à 2016. Fumeurs, selon le sexe, provinces et les territoires. Ottawa (ON): Statistiques Canada; 2017 [Consulté le 6 fév 2018]. https://www.statcan.gc.ca/tables-tableaux/ sum-som/l02/cst01/health74b-fra.htm

8. Beard M, Orlando JF, Kumar S. Overcoming the tyranny of distance: an audit of process and outcomes from a pilot telehealth spinal assessment clinic. J Telemed Telecare 2017 Sep;23(8):733-9. http://dx.doi. org/10.1177/1357633X16664851. PubMed (https://www.ncbi. nlm.nih.gov/entrez/query.fcgi?cmd=Retrieve\&db=PubMed\&li st_uids=27534822\&dopt=Abstract).

9. Pease C, Hutton B, Yazdi F, Wolfe D, Hamel C, Quach P et al. Efficacy and completion rates of rifapentine and isoniazid (3HP) compared to other treatment regimens for latent tuberculosis infection: a systematic review with network meta-analyses. BMC Infect Dis 2017 Apr;17(1):265. http:// dx.doi.org/10.1186/s12879-017-2377-x. PubMed (https:// www.ncbi.nlm.nih.gov/entrez/query.fcgi?cmd=Retrieve\&db=Pu bMed\&list_uids=28399802\&dopt=Abstract).

10. Oxlade O, Sugarman J, Alvarez GG, Pai M, Schwartzman K. Xpert@MTB/RIF for the Diagnosis of Tuberculosis in a Remote Arctic Setting: Impact on Cost and Time to Treatment Initiation. PLoS One 2016 Mar;11(3):e0150119. http://dx.doi. org/10.1371/journal.pone.0150119. PubMed (https://www. ncbi.nlm.nih.gov/entrez/query.fcgi?cmd=Retrieve\&db=PubMe d\&list_uids=26990299\&dopt=Abstract).

11. Affaires autochtones et du Nord Canada. Groupe de travail sur la turberculose - Document d'information [Consulté le 6 fév 2018]. https://www.canada.ca/en/indigenous-northernaffairs/news/2017/10/tuberculosis_taskforce.html

12. Inuit Tapiriit Kanatami. The National Representational Organization Protecting and Advancing the Rights and Interest of Inuit in Canada [Consulté le 6 fév 2018]. https://www.itk.ca/ 